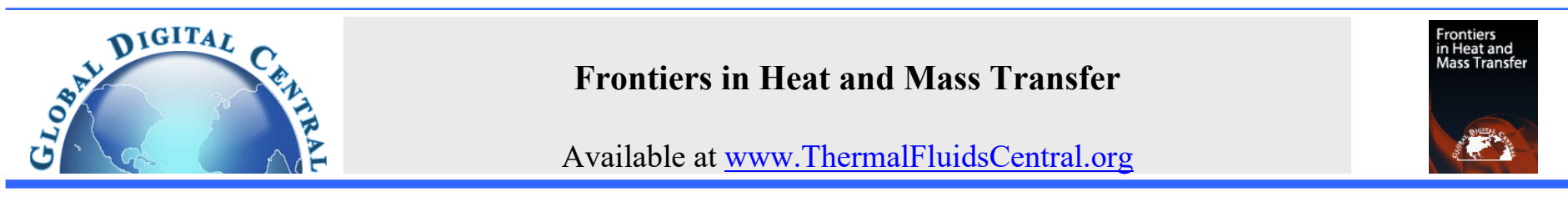

\title{
ENTROPY GENERATION AND TEMPERATURE GRADIENT HEAT SOURCE EFFECTS ON MHD COUETTE FLOW WITH PERMEABLE BASE IN THE PRESENCE OF VISCOUS AND JOULES DISSIPATION
}

\author{
K.S. Balamurugan ${ }^{\mathrm{a},}{ }^{*}$ N. Udaya Bhaskara Varma ${ }^{\mathrm{b}}$ and J.L. Ramaprasad ${ }^{\mathrm{c}}$ \\ ${ }^{a}$ Department of Mathematics, RVR \& JC College of Engineering, Guntur, Andhra Pradesh, 522019, India \\ ${ }^{b}$ Department of Basic Science \& Humanities, DNR College of Engineering \& Technology, Bhimavaram, Andhra Pradesh, 534202, India \\ ${ }^{c}$ Department of Mathematics, PB Siddhartha College of Arts and Science, Vijayawada, Andhra Pradesh, 520010, India
}

\begin{abstract}
In this paper the entropy generation and temperature gradient heat source effects on MHD couette flow with permeable base in the presence of thermal radiation, viscous and joule's dissipation is studied. An exact solution of governing equations has been attained in closed form. The influences of several parameters on the velocity and temperature profiles and entropy generation are analyzed through graphs. Bejan number for different values have been calculated and displayed pictorially. The skin friction coefficient and Nusselt number at channel walls are derived and discussed their behaviour through tables. The entropy generation increases with intensifying magnetic field or thermal radiation or empirical constant or Brinkman number.
\end{abstract}

Keywords: Entropy Generation, Temperature gradient, Couette flow, Bejan number.

\section{INTRODUCTION}

The interpretation of heat transfer and fluid flow in a porous channel has established considerable responsiveness during the last numerous decades due to their significance in an extensive range of engineering and biological situations such as problems of drainage, ground water hydrology and irrigation and also in processes of absorption and filtration in chemical engineering. Rapid development in science and technology has led to the expansion of cumulative number of flow divisor that includes the influence of the fluid flow in numerous geometries. In fluid mechanics couette flow is one of the basic flows wherever the fluid motion is induced by movement of the bounding surface. Flow formation examination in couette motion as foretold by classical fluid mechanics was presented by Schlichting and Gerstein (2000).

Everywhere the irreversible process in which time symmetry is ruined. By the theory of entropy the difference between reversible and irreversible process was first presented in thermodynamics. Entropy generation minimization examinations are vigorous for confirming best thermal systems in modern industrial and technological fields like electronic cooling, geothermal systems and heat exchangers. Entropy generation is squarely connected with thermodynamic irreversibility, entropy generation is the amount of the destruction of existing work of the system, the purpose of the dynamic factors motivating the entropy generation is significant in improvement the system performances. Bejan (1979) studied the entropy generation in forced convective heat transfer. Shohel Mahmud et al. (2005) examined the characteristics of thermal and entropy generation with viscous dissipation in a porous channel. Entropy generation with heat sink in a uniformly heated micro channel was analyzed by Abbassi (2007). Sheng Chen (2009) described an entropy generation owing to internal heat generation in turbulent natural convection. Mina Shahi et al. (2011) analyzed entropy generation of a Nano fluid owing to natural convection cooling. Ram Satish Kaluri and Tanmay Basak (2011) investigated entropy generation on thermal management during natural convection in porous square cavities with distributed heat sources. Analysis of convective heat transfer and entropy generation of $\mathrm{Al}_{2} \mathrm{O}_{3}$-water Nano fluid flow in a tangential heat sink was described by Amir Shalchi Tabrizi and Hamid Reza Seyf (2012). Basant et al. (2012) studied free convective flow of heat generation/absorbing fluid with ramped temperature past a vertical plate. Makinde (2012) presented the applications of thermodynamics second law to magnetic boundary layer flow past a plate by similarity and shooting technique. Chinyoka and Makinde (2013) analyzed the entropy generation rate in an unsteady porous channel flow with convective cooling and Navier slip. An entropy generation analysis of magnetic flow with buoyancy effects over a porous pipe was presented by Chinyoka et al. (2013). In wavy wall the effects of heat transfer and entropy generation of $\mathrm{H}_{2} \mathrm{O}$ based Nano fluid was presented by Ching Chang Cho et al. (2013). Entropy generation analysis of Nano fluid flow through the circular micro/mini channel heat sink was investigated by Sohel et al. (2013). Mansour et al. (2015) analyzed magneto hydrodynamic natural convection and entropy generation of a copper water Nano fluid with wall heat sink. Gamal and Rashed (2015) studied chemical entropy generation on unsteady fluid flow embedded in a porous medium. The effects of entropy generation and Bejan number on couette flow where lower plate is at rest and upper plate is moving with a uniform velocity is studied by Sukumar and Varma (2016). Baag et al (2017) examined entropy generation for viscoelastic flow through a stretching sheet with porous medium. Shantha Kumar Dutta and Arup Kumar Biswas (2018) studied the effects of permeability and the part of non-uniform heating in increasing the temperature and thermal energy and entropy generation minimization. Sudhakar and Balamurugan (2018) examined the effects of buoyancy force and Navier slip on the entropy with suction/injection

\footnotetext{
*Corresponding author. Email: muruganbalaks@gmail.com
} 
in a porous vertical channel. Arun and Jose Prakash (2018) investigated Heat transfer deterioration effects in rocket engine cooling channels. Zaidabadi Nezad and Mohammad Mehdi Keshtkar (2018) analyzed optimization of heat transfer from effective parameters on entropy generation in a square electronic package. Dharmaiah et al. (2019) studied the viscous dissipation effect and heat transfer characteristics of magneto flow through a moving inclined plate under the influence of aligned magnetic and thermal radiation. Tahar Taye (2019) investigated the entropy generation for free convection flow where the ground surface is subjected to uniform temperature and the other surface is subjected to constant temperature whereas the chimney wall is adiabatic by using finite volume method. Shyam Lal Yadav and Singh (2019) studied the effects of Darcy and viscous dissipation on free convective heat transfer couette flow in a partially filled saturated porous medium. The authors solved the equations of thermal energy corresponding to porous layer by the method of separation of variables. Hussein et al. (2019) analyzed the heat transfer and entropy generation of laminar flow. Hai-Dong Wang and, Zeng-Yuan Guo (2019) presented the physical essence of entropy in heat transfer.

The aim of this paper is to study the effects of entropy generation and temperature gradient heat source on MHD steady Couette flow bounded below by a permeable bed in the presence of thermal radiation, viscous dissipation and joules dissipation. The equations governing the flow are solved analytically and the impact of various flow parameters on velocity, temperature, entropy generation, Bejan number has been analyzed through graphs. The shear stress and rate of heat transfer coefficients at the channel walls are also derived and discussed their behavior through tables.

\section{FORMULATION OF THE PROBLEM}

Consider a steady flow of a Newtonian optically thick viscous incompressible fluid between two infinite horizontal parallel plates separated by a distance $H$. The upper plate moves with a uniform velocity $u_{0}$ in the fluid flow direction. A uniform magnetic field of strength $B$ is applied perpendicular to the fluid flow direction. A Cartesian coordinate system is chosen with $x$-axis along the lower stationary permeable surface and the $y$-axis is perpendicular to the plates.

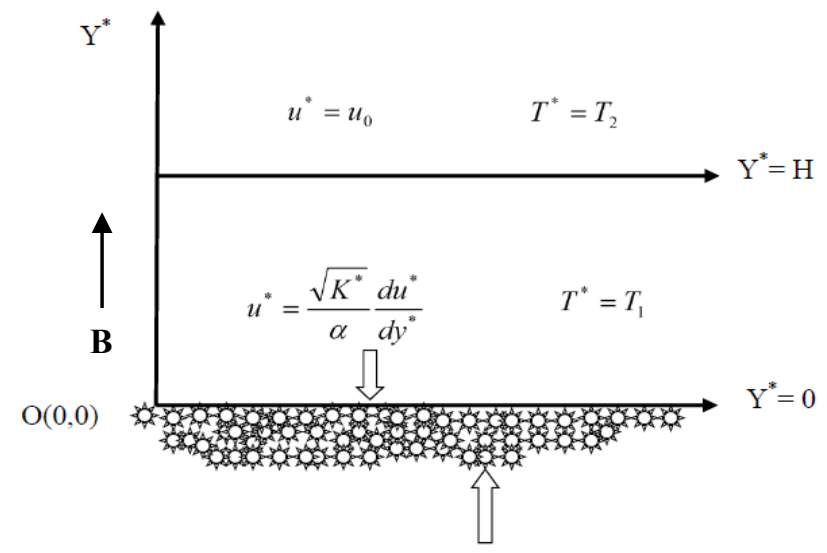

Fig. 1 Scheme diagram of the problem

The flow is considered under the following assumptions

1. The channel is long enough in $X$-direction so that all physical properties are independent of $x$ except pressure.

2. The flow is fully developed hydro dynamically and thermally.

3. Pressure and buoyancy forces are neglected.

4. Induced magnetic field and applied electric fields are neglected.
5. The temperatures of the lower and upper plates are $T_{1}$ and $T_{2}$ respectively, where $T_{2}>T_{1}$.

6. Viscous dissipation, joules dissipation and temperature gradient heat source effects are taken into account.

7. The radiative heat flux in the energy equation is assumed to follow Roseland approximation.

Under the above assumptions the governing momentum and energy equations for a steady flow of viscous incompressible fluid are

$$
\begin{aligned}
& \mu \frac{\partial^{2} u^{*}}{\partial y^{* 2}}-\sigma B^{2} u^{*}=0 \\
& k \frac{d^{2} T^{*}}{d y^{* 2}}+\mu\left(\frac{d u^{*}}{d y^{*}}\right)^{2}+\sigma B^{2} u^{*^{2}}-\frac{d q_{r}^{*}}{d y^{*}}+Q^{*} \frac{\partial}{\partial y^{*}}\left(T^{*}-T_{1}\right)=0
\end{aligned}
$$

The boundary conditions are

$$
\left.\begin{array}{ll}
y^{*}=H: & u^{*}=u_{0}, \quad T^{*}=T_{2} \\
y^{*}=0: & \frac{\partial u^{*}}{\partial y^{*}}=\frac{\alpha}{\sqrt{k}} u^{*}, \quad T^{*}=T_{1}
\end{array}\right\}
$$

where $\mu$ is dynamic viscosity, $k$ is thermal conductivity, $K^{*}$ is permeability, $T_{1}$ is the temperature of the lower boundary, $T_{2}$ is the temperature of the upper boundary, $H$ is the width of the channel. $B$ is magnetic field intensity, $\sigma$ is the electric conductivity, $Q^{*}$ is temperature gradient heat source parameter.

The radiation heat flux $q_{r}$ in the energy equation is assumed to follow Roseland approximation and is given by

$q_{r}=\frac{-4 \gamma^{*}}{3 \alpha^{*}} \frac{\partial T^{* 4}}{\partial y^{*}}$

Where $\gamma^{*}, \alpha^{*}$ are Stephan-Boltzmann constant and mean absorption constant respectively. We assume that the temperature difference within the fluid is sufficiently small so that $T^{* 4}$ may be expressed as a linear function of temperature about $T_{1}$ and omitting higher order terms to yield.

$T^{* 4} \cong 4 T_{1}^{3} T-3 T_{1}^{4}$

The non- dimensional quantities are

$u=\frac{u^{*}}{u_{0}}, \theta=\frac{T^{*}-T_{1}}{T_{2}-T_{1}}, y=\frac{y^{*}}{H}, K=\frac{K^{*}}{H^{2}}$

In view of equations (2.4)-(2.6) the equations (2.1) and (2.2) become

$$
\begin{aligned}
& \frac{d^{2} u}{d y^{2}}-M^{2} u=0 \\
& \left(1+\frac{4 N}{3}\right) \frac{d^{2} \theta}{d y^{2}}+B r\left(\frac{d u}{d y}\right)^{2}+B r M^{2} u^{2}+Q \frac{d \theta}{d y}=0
\end{aligned}
$$

The corresponding boundary conditions are

$$
\begin{aligned}
& u=\frac{\sqrt{K}}{\alpha} \frac{d u}{d y}, \quad \theta=0 \quad \text { at } \quad y=0 \\
& u=1, \quad \theta=1 \quad \text { at } \quad y=1
\end{aligned}
$$


Where $M^{2}=\frac{\sigma B^{2} H^{2}}{\mu}$ is Hartmann number, $B r=\frac{\mu u_{0}^{2}}{k\left(T_{2}-T_{1}\right)}$ is

Brinkman number, $Q=\frac{H}{k} Q^{*}$ is temperature gradient heat source parameter $(Q>0)$ and $N=\frac{4 \gamma^{*} T_{1}^{3}}{\alpha^{*} k}$ is Radiation parameter.

\subsection{Entropy Generation}

The local volumetric rate of entropy generation for a viscous incompressible conducting fluid in the presence of magnetic field, thermal radiation and viscous and joules dissipation is given by Woods (1975) and Arpaci (1987).

$E_{G}=\frac{k}{T_{1}^{2}}\left[\left(\frac{\partial T^{*}}{\partial y^{*}}\right)^{2}+\frac{16 \gamma^{*} T_{1}^{3}}{3 k \alpha^{*}}\left(\frac{\partial T^{*}}{\partial y^{*}}\right)^{2}\right]+\frac{\mu}{T_{1}}\left(\frac{\partial u^{*}}{\partial y^{*}}\right)^{2}+\frac{\sigma B^{2} u^{* 2}}{T_{1}}$

$E_{G_{0}}=\frac{k\left(T_{2}-T_{1}\right)^{2}}{H^{2} T_{1}^{2}}=($ characteristic entropy generation rate $)$

$\omega=\frac{T_{1}}{T_{2}-T_{1}}=($ characteristic temperature ratio $)$

The entropy generation number in terms of the dimensionless velocity and temperature is

$N S=\frac{E_{G}}{E_{G_{0}}}=\left(1+\frac{4}{3} N\right)\left(\frac{d \theta}{d y}\right)^{2}+B r \omega\left[\left(\frac{d u}{d y}\right)^{2}+M^{2} u^{2}\right]=H T I+F F I$

Where $H T I=\left(1+\frac{4}{3} N\right)\left(\frac{d \theta}{d y}\right)^{2}$ is the heat transfer irreversibility and

$F F I=B r \omega\left[\left(\frac{d u}{d y}\right)^{2}+M^{2} u^{2}\right]$ is the dissipative irreversibility

The Bejan number $B e$ is the pertinent irreversibility parameter and is defined as $B e=\frac{H T I}{N_{s}}$

Where $B e=1$ is the limit, at which heat transfer irreversibility dominates, $B e=0$ is the limit at which fluid friction irreversibility dominates and $B e=1 / 2$ implies that both of them contributes equally.

\section{MATHEMATICAL SOLUTION OF THE PROBLEM}

Solving the equations (7) and (8) subjected to the boundary conditions (9), the velocity and temperature distributions are obtained as follows

$$
\begin{aligned}
& u=c_{1} e^{M y}+c_{2} e^{-M y} \\
& \theta=c_{3}+c_{4} e^{-\frac{Q}{B} y}+c_{5} e^{2 M y}+c_{6} e^{-2 M y}
\end{aligned}
$$

The velocity and thermal regimes obtained are utilized in (11) to obtain the entropy regime.

\subsection{Skin Friction}

The skin friction across the channel's wall is given by

$$
\tau=\left(\frac{d u}{d y}\right)_{y=0, \quad y=1}
$$

Substituting (13) into equation (15), the skin friction across the channel's wall is given as

$$
\tau_{y=0}=c_{1} M-c_{2} M
$$

$\tau_{y=1}=c_{1} M e^{M}-c_{2} M e^{-M}$

\subsection{Nusselt Number}

The rate of heat transfer coefficient in terms of Nusselt number across the channel wall's is given by

$N u=\left(-\frac{\partial \theta}{\partial y}\right)_{y=0, y=1}$

Substituting (14) into equation (18), Nusselt number across the channel wall's is given by

$$
\begin{aligned}
& N u_{y=0}=\frac{Q}{B} c_{4}-2 M c_{5}+2 M c_{6} \\
& N u_{y=1}=\frac{Q}{B} c_{4} e^{-\frac{Q}{B}}-2 M c_{5} e^{2 M}+2 M c_{6} e^{-2 M}
\end{aligned}
$$

Where

$$
\begin{aligned}
& c_{1}=\frac{M+\frac{\alpha}{\sqrt{K}}}{\left(M+\frac{\alpha}{\sqrt{K}}\right) e^{M}+\left(M-\frac{\alpha}{\sqrt{K}}\right) e^{-M}}, \quad B=1+\frac{4 N}{3} \\
& c_{2}=\frac{M-\frac{\alpha}{\sqrt{K}}}{\left(M+\frac{\alpha}{\sqrt{K}}\right) e^{M}+\left(M-\frac{\alpha}{\sqrt{K}}\right) e^{-M}}, \quad c_{3}=-c_{4}-c_{5}-c_{6}, \\
& c_{4}=\frac{c_{5}\left(e^{2 M}-1\right)+c_{6}\left(e^{-2 M}-1\right)-1}{1-e^{-\frac{Q}{B}},} c_{5}=\frac{-2 B r c_{1}^{2} M^{2}}{\left(4 B M^{2}+2 M Q\right)}, \\
& c_{6}=\frac{-2 B r c_{2}^{2} M^{2}}{\left(4 B M^{2}-2 M Q\right)}
\end{aligned}
$$

\section{RESULTS AND DSICUSSION}

The effects of different parameters such as Hartmann number $M$, Brinkman number $B r$, radiation parameter $N$, permeability parameter $\mathrm{K}$, dimensionless empirical constant $\alpha$, characteristic temperature ration $\omega$ and temperature gradient heat source parameter $Q$, are examined on the velocity, temperature, Bejan number and entropy generation. The closed form solution obtained for velocity and temperature are employed to compute the entropy generation. The findings are depicted through graphs.

Figures 2 and 3 illustrate the variations of velocity $u$ and temperature distribution $\theta$ for different values of empirical constant $\alpha$ and permeability parameter $\mathrm{K}$. It is observed that the velocity decreases with increasing empirical constant but it improves the temperature distribution as wells as the thermal boundary layer thickness. The increasing $K$ reduces fluid friction with channel wall and velocity enhances which is perceptible from figure 2. Physically, this means that the porous medium on the boundary layer growth is significant due to increase in the permeability of the porous medium leads to the rise in the flow of fluid through it when the holes of the porous medium become large, the resistance of the medium may be neglected. But the opposite behavior in temperature distribution. The variations in temperature $\theta$ for different values of Brinkman number $B r$, temperature gradient heat source parameter $Q$ and radiation parameter $\mathrm{N}$ are presented in Figure 4. It is noticed that the temperature $\theta$ is increases with increasing Brinkman number $\mathrm{Br}$ and temperature gradient heat source parameter $Q$. Due to the improvement in Brinkman number results in enhanced convective transport. The result of $Q$ is very much significant for the flow where heat transfer is given prime important whereas rising values of radiation parameter depreciate the fluid temperature. 


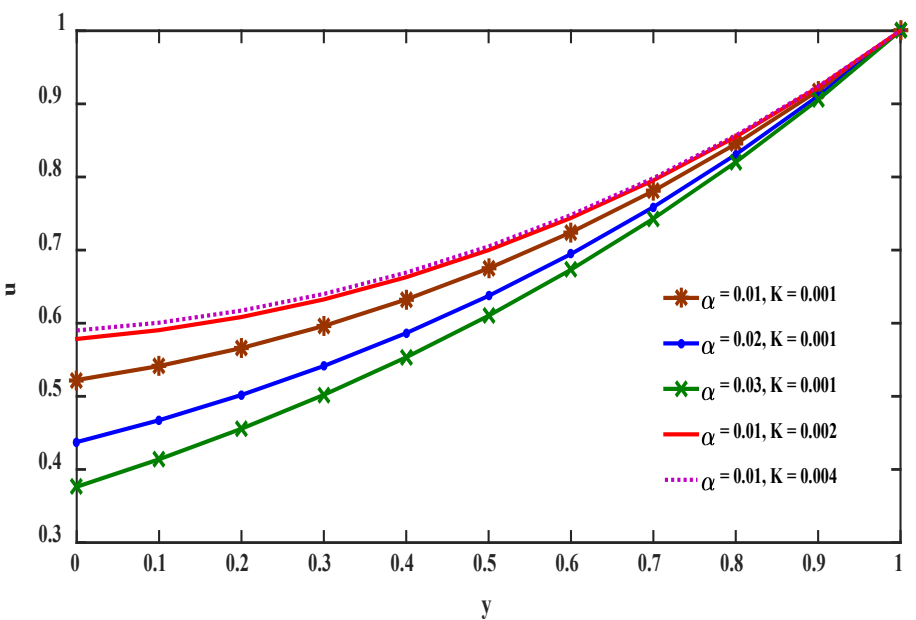

Fig. 2 Velocity profiles for different values of non-dimensional empirical constant $\alpha$ and permeability parameter $\mathrm{K}$ when $\mathrm{N}=1$, $\mathrm{Br}=10, \mathrm{Q}=0.1$ and $\mathrm{M}=1$.

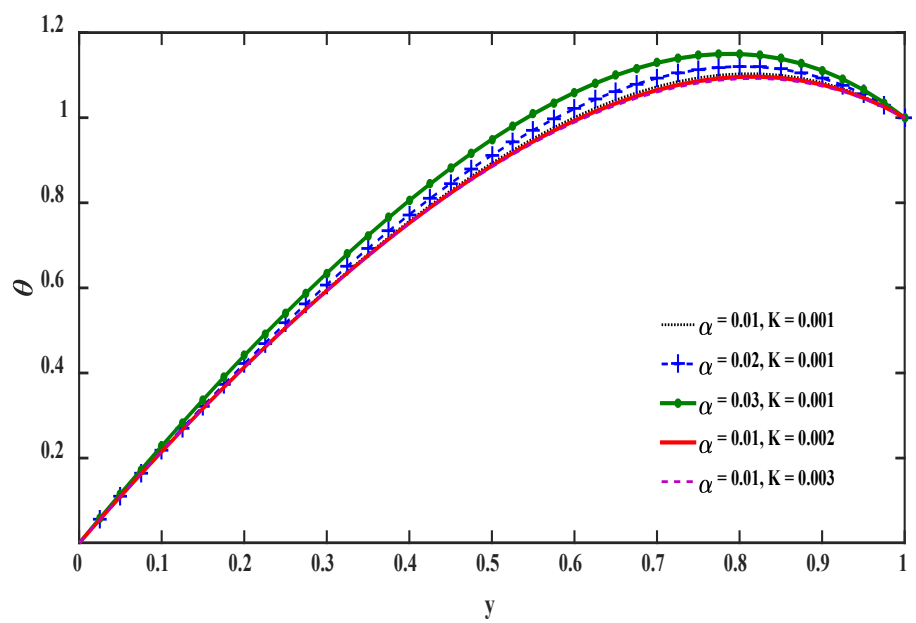

Fig. 3 Temperature profiles for different values of non-dimensional empirical constant $\alpha$ and permeability parameter $\mathrm{K}$ when $\mathrm{N}=1$, $\mathrm{Br}=10, \mathrm{Q}=0.1$ and $\mathrm{M}=1$.

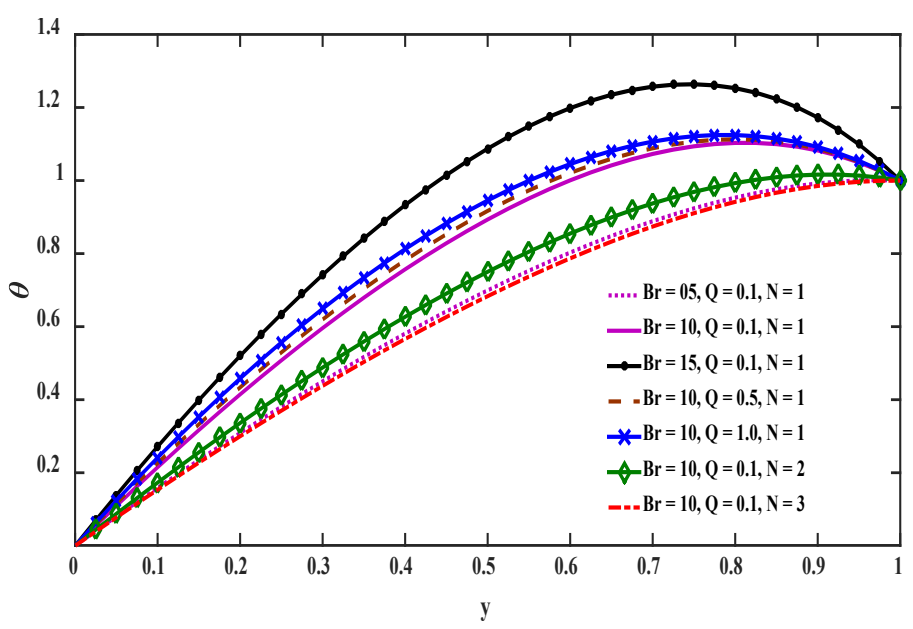

Fig. 4 Temperature profiles for different values of Brinkman number $\mathrm{Br}$, temperature gradient heat source parameter $\mathrm{Q}$ and radiation parameter $\mathrm{N}$ When $\alpha=0.01, \mathrm{~K}=0.001$ and $\mathrm{M}=1$

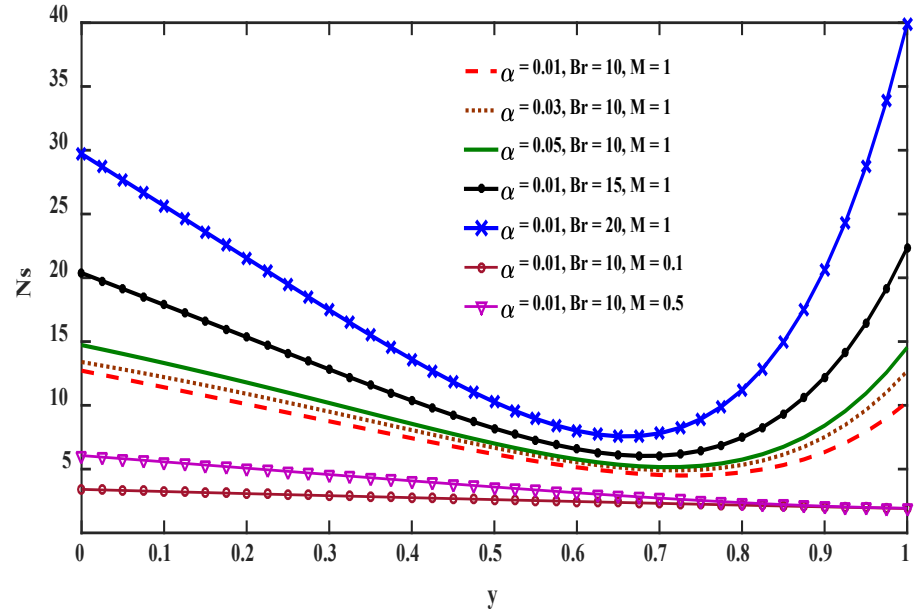

Fig. $5 \mathrm{Ns}$ for different values of empirical constant $\alpha$, brinkman number $\mathrm{Br}$ and Hartman number $\mathrm{M}$ when $\mathrm{K}=0.001, \mathrm{Q}=0.1$, $\mathrm{N}=1, \omega=0.4$.

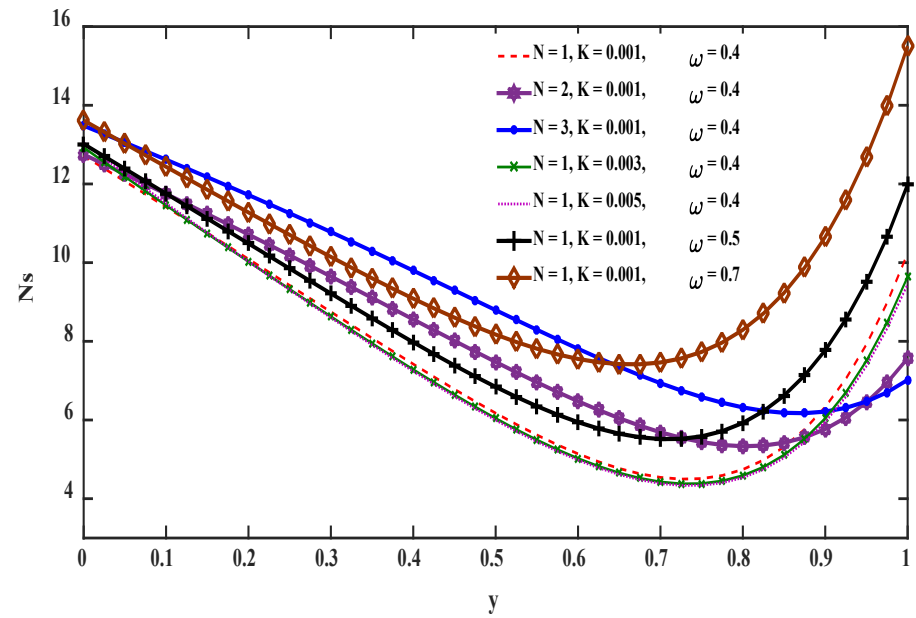

Fig. $6 \mathrm{Ns}$ for different values of radiation parameter N, permeability parameter $\mathrm{K}$ and characteristic temperature ration $\omega$ when $\alpha=0.01, \mathrm{Br}=10, \mathrm{M}=1, \mathrm{Q}=0.1$.

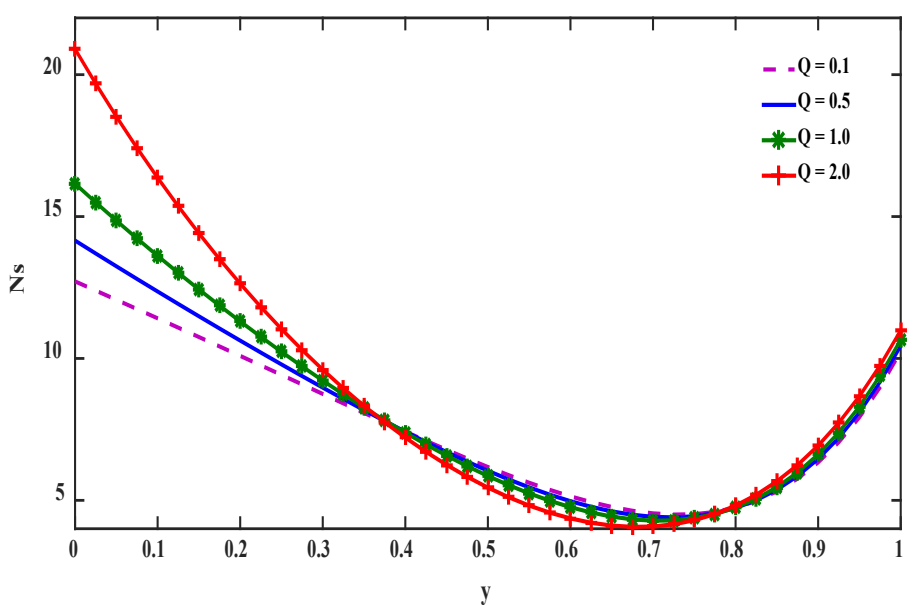

Fig. $7 \mathrm{Ns}$ for different values of temperature gradient heat source parameter Q when $\alpha=0.01, \mathrm{Br}=10, \mathrm{M}=1, \mathrm{~K}=0.001, \mathrm{~N}=1$ and $\omega=0.4$. 


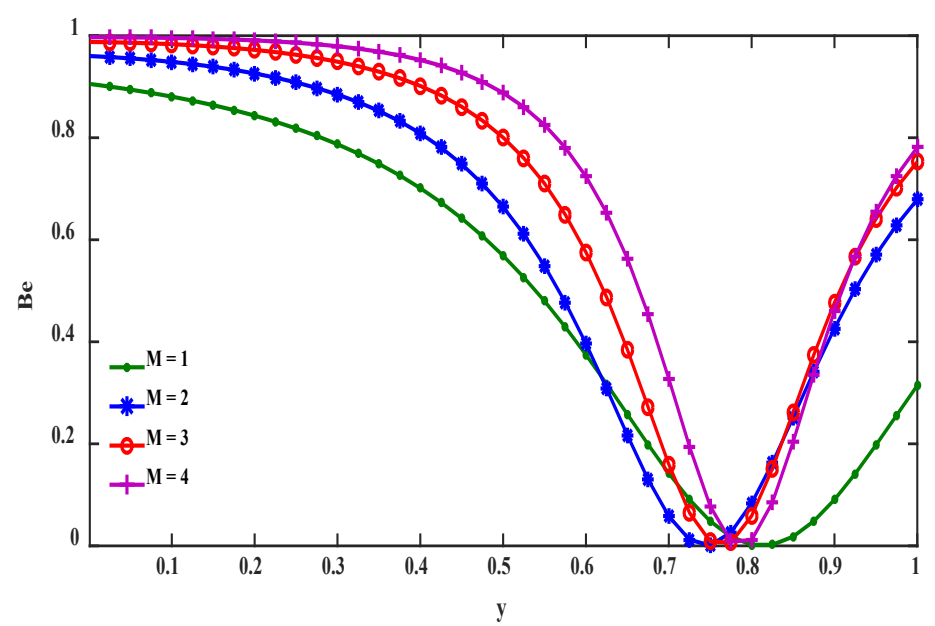

Fig. 8 Bejan number for different values of Hartman number $M$ when $\mathrm{N}=1, \mathrm{~K}=0.001, \omega=0.4, \alpha=0.01, \mathrm{Br}=10$, and $\mathrm{Q}=0.1$.

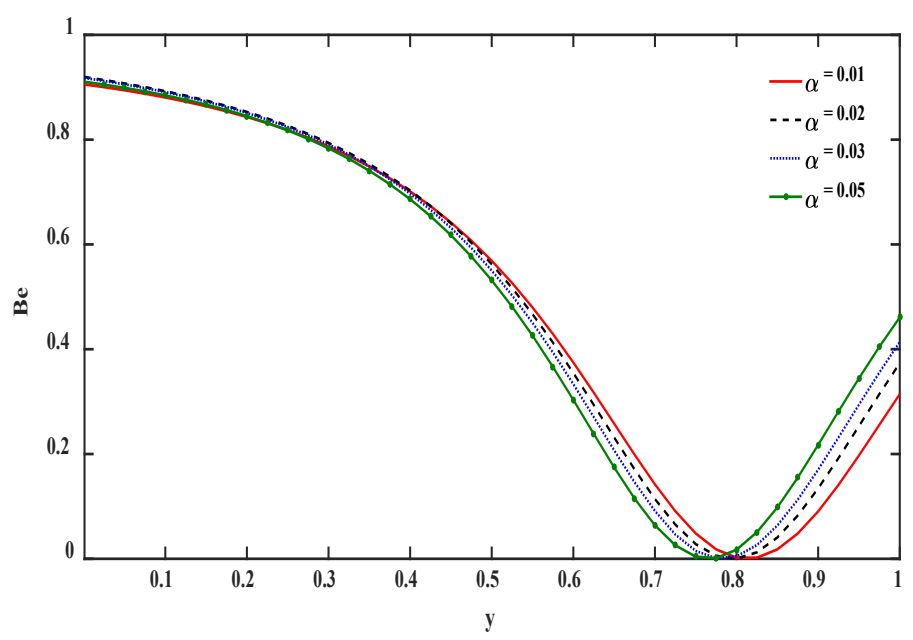

Fig. 9 Bejan number for different values of empirical constant $\alpha$ when $\mathrm{M}=1, \mathrm{~N}=1, \mathrm{~K}=0.001, \omega=0.4, \mathrm{Br}=10$, and $\mathrm{Q}=0.1$.

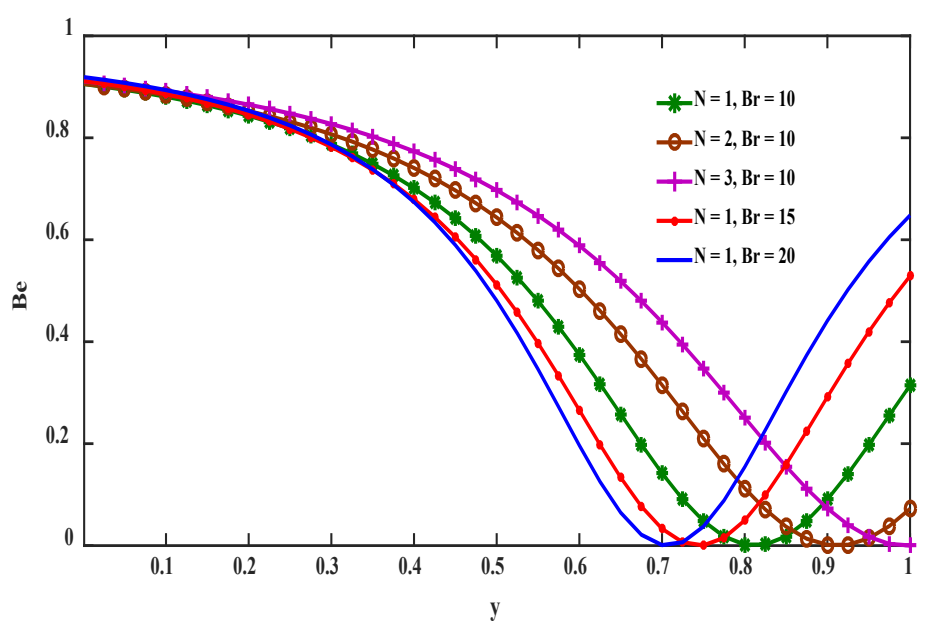

Fig. 10 Bejan number for different values of radiation parameter $\mathrm{N}$ and Brinkman number $\mathrm{Br}$ when $\alpha=0.01, \mathrm{M}=1, \mathrm{~K}=0.001, \omega=0.4$, and $\mathrm{Q}=0.1$.

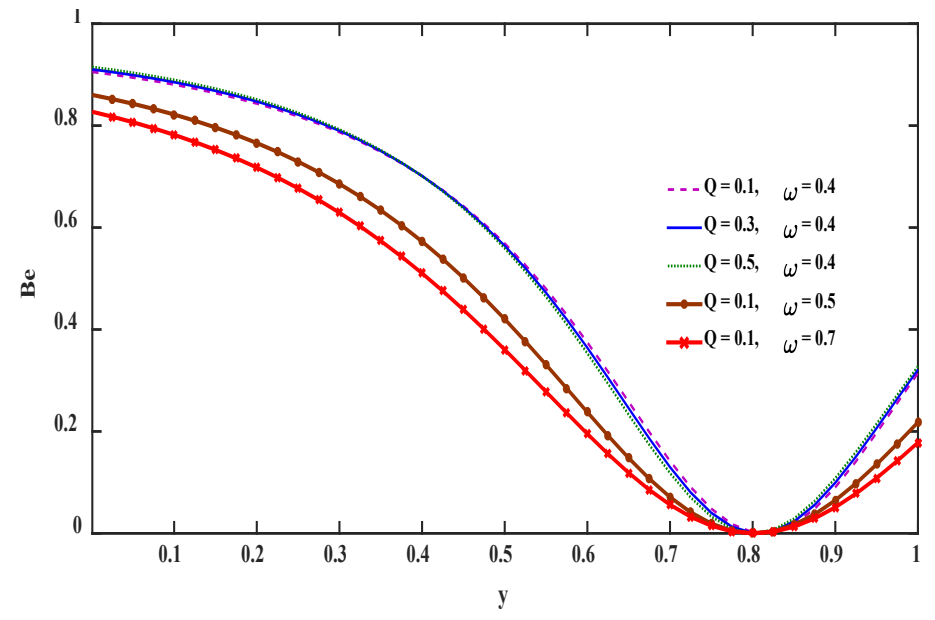

Fig. 11 Bejan number for different values of temperature gradient heat source parameter $\mathrm{Q}$ and characteristic temperature ration $\omega$ when $\alpha=0.01, \mathrm{M}=1, \mathrm{~N}=1, \mathrm{~K}=0.001$ and $\mathrm{Br}=10$.

The variation in entropy generation number $N s$ for different values of Hartmann number $M$, dimensionless empirical constant $\alpha$, Brinkman number $B r$, temperature gradient heat source parameter $Q$, permeability parameter $\mathrm{K}$, Radiation parameter $N$ and characteristic temperature ratio $\omega$ are shown in Figures 5-7. From figures $5 \& 6$ it is noticed that the entropy generation number increases with increasing values of $\mathrm{M}$ or $B r$ or $\alpha$ or $N$ or $\omega$. But entropy generation decreases due to porosity parameter K. From figure 7, by increasing temperature gradient heat source parameter $Q$ entropy generation falls in the lower and upper part of the channel but in the middle region the reverse effect is witnessed.

The variation in Bejan number for different values of Hartmann number $\mathrm{M}$, dimensionless empirical constant $\alpha$, Radiation parameter $\mathrm{N}$, Brinkman number $\mathrm{Br}$, temperature gradient heat source parameter $Q$ and characteristic temperature ratio $\omega$ are shown in figures 8-11. From figure 8 it is noticed that the Bejan number increases with increasing values of Hartmann number. From figure 9 it is noticed that dimensionless empirical constant has no much effect in the lower part of the channel, but in the middle of the channel Bejan number decreases with increasing values of whereas it rises in the in the upper part of the channel. Influences of radiation parameter, Brinkman number, temperature gradient heat source parameter and characteristic temperature ratio on Bejan number are shown in figures $10 \& 11$. Figures are reported that the Be decreases with increasing values of $Q$ and $\omega$.But opposite phenomenon is reported in case of radiation parameter N. Also Be decays in the lower part and middle of the channel whereas it increases in the upper part of the channel with the increasing values of $\mathrm{Br}$.

Table 1 elucidates that the Nusselt number decreases with an increase in heat source parameter $Q$ and Brinkman number $\mathrm{Br}$ at the wall $\mathrm{y}=0$ but this trend is reversed at the wall $y=1$. It is evident to conclude that the heat transfer rate is high at upper plate when compared with lower plate.

Table 1 Numerical values of Nusselt number for different values of Brinkman number $B r$ and heat source parameter $Q$ with $\alpha=0.01, \mathrm{M}=1$, $\mathrm{N}=1, \mathrm{~K}=0.001$ and $y=0,1$

\begin{tabular}{|c|c|c|c|c|c|c|}
\hline \multirow{2}{*}{$B r \backslash Q$} & \multicolumn{3}{|c|}{$-\theta^{1}(0)$} & \multicolumn{3}{c|}{$-\theta^{1}(1)$} \\
\cline { 2 - 7 } & 0.1 & 0.5 & 1 & 0.1 & 0.5 & 1 \\
\hline 1 & -1.1416 & -1.2355 & -1.3598 & -0.7634 & -0.6859 & -0.5959 \\
\hline 5 & -1.6216 & -1.7336 & -1.8807 & 0.0978 & 0.1572 & 0.2244 \\
\hline 10 & -2.2215 & -2.3562 & -2.5318 & 1.1743 & 1.2111 & 1.2498 \\
\hline 15 & -2.8215 & -2.9788 & -3.1829 & 2.2509 & 2.2650 & 2.2752 \\
\hline
\end{tabular}




\section{CONCLUSIONS}

The studies of Entropy generation minimization are significant role for confirming optimal thermal methods in modern technological and industrial fields like heat exchangers, geothermal systems, electronic cooling,. Entropy generation, heat transfer, thermal radiation and temperature gradient heat source effects on a steady Couette flow bounded below by a permeable bed has been studied and analyzed. Based on the results obtained the following conclusions are made.

$>$ The Brinkman number enhances the thermal boundary layer thickness.

$>$ The fluid velocity can be enhanced and reduced temperature by increasing porosity suitably.

$>$ Temperature $\theta$ increases with increasing temperature gradient heat source parameter $Q$. But thermal radiation depreciates the fluid temperature.

$>$ The entropy generation number increases with increasing values of $\mathrm{M}$ or $\alpha$ or $\mathrm{Br}$ or $\mathrm{N}$ or $\omega$. Reverse trend is observed due to $\mathrm{K}$.

$>$ By increasing temperature gradient heat source parameter $Q$ the entropy generation falls in the lower and upper part of the channel but in the middle region the reverse effect is witnessed.

$>$ Bejan number $B e$ increases with increasing values of $\mathrm{M}$ or $\mathrm{N}$ and decreases with increasing values of $\omega$ or $Q$.

$>$ Bejan number decrease in the region $0 \leq \mathrm{y} \leq 0.8$ with increasing and reverse phenomena is observed in the region $0.8 \leq y \leq 1$.

$>$ Nusselt number decreases with an increase in $Q$ and $B r$ at the wall $\mathrm{y}=0$ but this trend is reversed at the wall $y=1$.

$>$ The heat transfer rate is high at upper plate when compared with lower plate.

\section{NOMENCLATURE}

$B \quad$ magnetic coefficient

$x^{*}, y^{*} \quad$ Coordinate system

$k \quad$ thermal conductivity

M magnetic parameter

$q_{r} \quad$ heat flux

$N \quad$ radiation parameter

$\mu \quad$ Dynamic viscosity

$T_{1}, T_{2} \quad$ Temperatures at lower and upper boundary

$u \quad$ dimensionless velocity

$H \quad$ width of the channel

$Q \quad$ temperature gradient heat source parameter

$K \quad$ permeability parameter

$\mathrm{Br} \quad$ Brinkman number

$\tau \quad$ Skin friction coefficient

$\mathrm{Nu} \quad$ Nusselt number

$u_{0} \quad$ uniform velocity

$N_{S} \quad$ Entropy generation number

Be Bejan number

Greek Symbols

$\theta \quad$ fluid temperature

$\rho \quad$ density

$\sigma \quad$ electric conductivity

$\omega \quad$ Characteristic temperature ratio

Superscripts

* Dimensional parameters

\section{REFERENCES}

Schlichting, H., and Gerstein, K., 2000, "Boundary layer theory": $8^{\text {th }}$ revised and enlarged edition (English), Springer-Verlag, New York.
Bejan, A., 1979. "A study of entropy generation in fundamental convective heat transfer", Journal of Heat Transfer, 101(4), 718-725. https://doi.org/10.1115/1.3451063.

Shohel Mahmud and Roydon Andrew Fraser., 2005, "Flow, thermal and entropy generation characteristics inside a porous channel with viscous dissipation", International Journal of thermal Sciences, 44(1), 21-32.

https://doi.org/10.1016/j.ijthermalsci.2004.05.001.

Abbasi, H., 2007, "Entropy generation analysis in a uniformly heated micro channel heat sink". Energy, 32(10), 1932-1947.

https://doi.org/10.1016/j.energy.2007.02.007.

Sheng Chen and Manfred Krafezyk, 2009, "Entropy generation in turbulent natural convection due to internal heat generation", International Journal of Thermal Sciences, 48(10), 1978-1987.

https://doi.org/10.1016/j.ijthermalsci.2009.02.012.

Mina Shahi, Amir Houshang Mahmoudi and Abbas Honarbakhsh Raouf, 2011, "Entropy generation due to natural convection cooling of a Nano fluid", International Communications in Heat and Mass Transfer, 38(7), 972-983.

https://doi.org/10.1016/j.icheatmasstransfer.2011.04.008.

Ram Satish Kaluri, and Tanmay Basak., 2011, "Role of entropy generation on thermal management during natural convection in porous square cavities with distributed heat sources", Chemical Engineering Science, 66(10), 2124-2140.

https://doi.org/10.1016/j.ces.2011.02.009.

Amir Shalchi Tabirzi and Hamid Reza Seyf, 2012, "Analysis of entropy generation and convective heat transfer $\mathrm{Al}_{2} \mathrm{O}_{3}$ Nano fluid flow in a tangential micro heat sink", International Journal of Heat and Mass Transfer, 55 (15-16), 4366-4375. https://doi.org/10.1016/j.ijheatmasstrasnfer.2012.04.005.

Basant K. Jha, Ahmad K. Samaila and Abiodun O. Ajibade., 2012, "Natural convection flow of heat generation/absorption fluid near a vertical plate with ramped temperature", Journal of Encapsulation and Absorption Sciences, 2, 61-68.

https://dx.doi.org/10.4236/jeas.2012.24009p.

Makinde, O.D., 2012, "Entropy analysis for MHD boundary layer flow and heat transfer over a flat plate with a convective surface boundary condition", International Journal of Exergy, 10(2), 142-154. https://doi.org/10.1504/IJEX.2012.045862

Chinyoka, T., and Makinde, O.D., 2013, "Analysis of entropy generation rate in an unsteady porous channel flow with Navier slip and convective cooling", Entropy, 15(6), 2081- 2099.

https://doi.org/10.3390/e15062081.

Chinyoka, T., Makinde, O.D and Adetayo Samuel Eegunjobi, 2013, "Entropy analysis of unsteady magnetic flow through a porous pipe with buoyancy effects", Journal of Porous Media. 16(9), 823-836. http:/dx.org/10.1615/JPorMedia.v16.i9.40.

Ching Chang Cho, Chich Li Chen and Chao's Kuang Chen, 2013, "Natural convection heat transfer and entropy generation in wavy wall enclosure containing water based Nano fluid", International Journal of Heat and Mass Transfer, 61, 749-758.

https://doi.org/10.1016/j.ijheatmasstransfer.2013.02.044.

Sohel, M.R., Saidur, R., Hassan, N.H., Elias, M.M., Khaleduzzaman, S.S., and Mahbubul, I.M., 2013, "Analysis of entropy generation using nanofluid flow through circular micro channel and mini channel heat sink", International Communications in Heat and Mass Transfer, 46, $85-91$

https://doi.org/10.1016/j.icheatmasstransfer.2013.05.011.

Mansour, M.A., Chamka Ali J., and Bakier, A.Y., 2015, "Magneto hydrodynamic natural convection and entropy generation of a $\mathrm{Cu}$ water 
Nano fluid in a cavity with wall mounted heat source/sink", Journal of Nano Fluid, 4(2), 254-269.

https://doi.org/10.1166/jon.2015.1147.

Gamal M., and Abdel Rahman Rashed, 2015, "Chemical entropy generation and MHD effects on the unsteady heat and fluid flow through a porous medium". Journal of Applied Mathematics, 2016, $1-9$.

https://doi.org/10.1155/2016/1748312.

Sukumar M., and Varma, S.V.K., 2016, "Entropy generation and temperature dependent heat source effects on MHD couette flow with permeable base in the presence of radiation and viscous dissipation", Middle East Journal of Scientific Research, 24(8), 2577-2588.

https://doi.org/10.5829/idosi.mejsr.2016.24.08.23830.

Baag, S., Mishra, S.R., Dash G.C., and Acharya, M.R., 2017, "Entropy generation analysis for Visco elastic MHD flow over a stretching sheet embedded in a porous medium", Ain Shams Engineering Journal, 8(4), 623-632.

https://doi.org/10.1016/j.asej.2015.10.017.

Shantha Kumar Dutta and Arup Kumar Biswas, 2018, "Entropy generation due to natural convection with non-uniform heating porous quadrantal enclosure a numerical study", Frontiers in Heat and Mass Transfer, 10, 8, 1-12.

http://dx.doi.org/10.5098/hmt.10.8.

Sudhakar, M and Balamurugan, K.S., 2018, "Entropy generation analysis in a vertical porous channel with Navier slip in the presence of viscous dissipation and heat source", International Journal of Mechanical and Production Engineering Research and Development, 8(5), 261-270.

https;//doi.org/10.24247/ijmperdoct201829.

Arun, M and Jose Prakash, M, 2018, "Heat transfer deterioration effects of cryogenic methane in rocket engine cooling channels", Frontiers in Heat and Mass Transfer, 11, 9, 1-10.

http://dx.doi.org/10.5098/hmt.11.9.
Saeed Zaidabadi Nezad and Mohammad Mehdi Keshtkar, 2018, "Investigation of effective parameters on entropy generation in a square electronic package", Frontiers in Heat and Mass Transfer, 10, 11, 1-7. http://dx.doi.org/10.5098/hmt.10.11.

Dharmaiah, G., Chamkha, Ali, J., Vedavathi, N and Balamurugan, K.S, 2019, "Viscous dissipation effect on transient aligned magnetic free convective flow past an inclined moving plate", Frontiers in Heat and Mass Transfer, 12, 17, 1-11.

http://dx.doi.org/10.5098/hmt.12.17.

Tahar Taye., 2019, "Entropy generation analysis of convective airflow in a solar updraft tower power plant", Heat Transfer Asian Research, , 48(18), 3885-3901

https://doi.org/10.1002/htj.21573.

Shyam Lal Yadav and Singh A.K., 2019, "Analysis of entropy generation in a horizontal channel containing fluid and porous layers", Journal of Porous Media, 22(10), 1259-1271. https://doi.org/10.1615/JPorMedia.2019025809.

Hussein M Jassim, Farooq H.A., Qusay R Al-Amir, Hameed K Hamzah, Salwan Obaid Waheed Khafaji, 2019, "Entropy generation analysis of a natural convection inside a sinusoidal enclosure with different shapes of cylinders", Frontiers in Heat and Mass Transfer, 12, 22, 1-9.

http://dx.doi.org/10.5098/hmt.12.22.

Hai-Dong Wang and Zeng-Yuan Guo, 2019, "Physical Heat Transfer", Frontiers in Heat and Mass Transfer, 13, 20, 1-12.

http://dx.doi.org/10.5098/hmt.13.20.

Woods, L C., 1975, "Thermodynamics of fluid systems". Oxford, UK: Oxford University Press.

Arpaci, V.S., 1987, "Radiative entropy production lost heat into entropy", International Journal of Heat and Mass Transfer, 30 (10), $2115-2123$.

https://doi.org/10.1016/0017-9310(87)90090-1. 